\title{
NOTE ON THE HARMONIC OSCILLATOR IN GENERAL RELATIVITY
}

\author{
N. W. TAYLOR
}

(received 12 December 1960, revised 23 January 1961)

The theory of relativity shows that the times measured by two observers will in general be different if they are in relative motion, so that their respective times between any two coincidences will differ. Bergmann [1] has investigated the problem of a particle moving in a small simple harmonic motion in a static gravitational field, and has found that the time difference for this particle and an observer at rest becomes zero whenever the particle passes through the centre and limits of its swing. This problem will now be dealt with in a different manner, using Schwarzschild's interior solution of the gravitational equations. The exterior solution for a point mass is not suitable in the present case, due to the singularity of the field at a point in the path of the particle.

Møller [2] gives Schwarzschild's interior solution in the form

$$
d s^{2}=\frac{d r^{2}}{1-r^{2} / R^{2}}+r^{2}\left(d \theta^{2}+\sin ^{2} \theta \cdot d \phi^{2}\right)-\left\{A-B\left(1-r^{2} / R^{2}\right)^{\frac{1}{2}}\right\} c^{2} d t^{2} .
$$

Making the substitutions $r=R \sin (\rho / R)$ and $t=\tau /(A-B)$, and using the time-like instead of the space-like form, this becomes

$$
d s^{2}=-d \rho^{2}-R^{2} \sin ^{2} \frac{\rho}{R} \cdot\left(d \theta^{2}+\sin ^{2} \theta \cdot d \phi^{2}\right)+\left\{\frac{A-B \cos \frac{\rho}{R}}{A-B}\right\}^{2} c^{2} d \tau^{2} .
$$

The coordinate $\rho$ determines the distance from the origin and so, for a motion which does not depart too far from the origin, the metric can be taken in the approximate form

$$
d s^{2}=-d \rho^{2}-\rho^{2}\left(d \theta^{2}+\sin ^{2} \theta \cdot d \phi^{2}\right)+\gamma c^{2} d \tau^{2}
$$

where

$$
\gamma=1+\eta \rho^{2},
$$

$\eta$ being a constant which is small for ordinary fields.

The equations of motion of a particle are the geodesic equations 


$$
\frac{d^{2} x^{\varepsilon}}{d s^{2}}+\left\{\begin{array}{c}
\varepsilon \\
\mu \nu
\end{array}\right\} \frac{d x^{\mu}}{d s} \frac{d x^{\nu}}{d s}=0 .
$$

Evaluating the Christoffel symbols for a static spherically symmetric system, it is seen that the $\theta$ - and $\phi$-components of the equations (3) are satisfied by $\theta=\pi / 2$ and $\phi=$ constant. The $\tau$-component is

$$
\frac{d^{2} \tau}{d s^{2}}+\frac{1}{\gamma} \cdot \frac{d \gamma}{d \rho} \cdot \frac{d \rho}{d s} \frac{d \tau}{d s}=0
$$

which can be integrated to give

$$
\frac{d \tau}{d s}=\frac{1}{c \alpha \gamma} \text {. }
$$

Instead of the $\rho$-component of (3), it is more convenient to take the expression (1). In the radial type of motion under consideration, this reduces to

$$
d s^{2}=-d \rho^{2}+\gamma c^{2} d \tau^{2} .
$$

Eliminating $d s$ from (4) and (5),

$$
\left(\frac{d \rho}{d \tau}\right)^{2}=c^{2}\left(\gamma-\alpha^{2} \gamma^{2}\right)
$$

If the field is not too strong, $\eta^{2}$ is negligible, and so, approximately,

$$
\left(\frac{d \rho}{d \tau}\right)^{2}=c^{2}\left(1-\alpha^{2}\right)-c^{2}\left(2 \alpha^{2}-1\right) \eta \varphi^{2} .
$$

This is the equation of a simple harmonic motion. It shows that $\alpha$ is less than unity by an amount of the order of $v^{2} / 2 c^{2}$ where $v$ is the maximum velocity of the motion. The equation can be integrated to the form

$$
\rho=a \sin \omega \tau,
$$

where

$$
\omega=c\left\{\eta\left(2 \alpha^{2}-1\right)\right\}^{3}
$$

and

$$
a=\left\{\frac{1-\alpha^{2}}{\eta\left(2 \alpha^{2}-1\right)}\right\}^{t}
$$

Let $R$ be an observer remaining at the origin, and $M$ an observer.on the moving particle. Times measured by the observers on any suitable physical apparatus are given by measurements of interval, $\int d s / c$. So, from (1) and (2), the time measured by $R$ between the initial coincidence and the event that, in $R$ 's estimation, $M$ is at a given point in space is

$$
\int_{0}^{T} d \tau
$$


where $T$ is the time coordinate of this event. The corresponding time measurement for $M$ is, by (4).

$$
\int_{0}^{T} \alpha \gamma d \tau
$$

the range of integration being the same since the coordinate system is used to specify events, irrespective of any observer. Using (2) and (6), the difference in these two times is

or

$$
\Delta=T-\int_{0}^{T} \alpha\left(1+\eta a^{2} \sin ^{2} \omega \tau\right) d \tau,
$$

$$
\Delta=\left(1-\alpha-\frac{1}{2} \alpha \eta a^{2}\right) T+\frac{\alpha \eta a^{2}}{4 \omega} \sin 2 \omega T .
$$

Using (8),

$$
\Delta=-\frac{(1-\alpha)^{2}(3 \alpha+2) T}{2\left(2 \alpha^{2}-1\right)}+\frac{\alpha\left(1-\alpha^{2}\right)}{4 \omega\left(2 \alpha^{2}-1\right)} \sin 2 \omega T .
$$

Since $\alpha$ is less than unity by an amount of the order of $v^{2} / 2 c^{2}$ where $v$ is the maximum velocity of the motion, the first term on the right hand side of the equation (9) is of the order of $\left(v^{4} / c^{4}\right) T$. Hence

$$
\Delta=O\left(\frac{v^{4}}{c^{4}}\right) T+\frac{1-\alpha}{2 \omega} \sin 2 \omega T .
$$

The second term on the right hand side becomes zero when, in the estimation of the observer at rest, the moving particle is at the centre and limits of its path. For a similar motion produced by a force other than gravity so that special relativity could be used, the time difference would be of the order $\left(v^{2} / c^{2}\right) T$. Hence, in the case of harmonic motion under gravity, the time difference is comparatively negligible whenever the moving particle is at the centre and limits of its path. This agrees with the result of Bergmann.

\section{References}

[1] Bergmann, O., "Einige Bemerkungen zum Uhrenparadox", Acta Physica Austriaca, XI (1957), 377-389.

[2] Moller, C., The Theory of Relativity, Oxford, (1952), p. 330.

Department of Mathematics,

University of New England, Armidale, New South Wales. 